\title{
A Ka-band GaAs MMIC quadrupler with high dynamic range and efficient harmonics rejection
}

\author{
Yuehong Dong ${ }^{1}$, Yuehang $\mathrm{Xu}^{1 \mathrm{a})}$, Junheng Huang ${ }^{2}$, Ying $\mathrm{Ju}^{3}$, \\ Lei $\mathrm{He}^{3}$, Tiedi Zhang ${ }^{1}$, Ruimin $\mathrm{Xu}^{1}$, and Bo Yan ${ }^{1}$ \\ ${ }^{1}$ School of Electronic Engineering, University of Electronic Science and \\ Technology of China, Chengdu 611731, People's Republic of China \\ ${ }^{2}$ IC Valley Microelectronics., Ltd., Hefei, Anhui 230088, People's Republic of China \\ 3 Jiuzhou Electric Group Co., Ltd., \\ Mianyang, Sichuan 621000, People's Republic of China
}

a)yuehangxu@uestc.edu.cn

Abstract: We report a Ka-band monolithic frequency quadrupler with high dynamic range and harmonics efficient rejection based on $0.15 \mu \mathrm{m} \mathrm{GaAs}$ PHEMT process in this letter. The quadrupler is constructed a drive power amplifier, a one-stage active quadrupling part, a high-pass filter, and a threestage power amplifier of the fourth harmonic. Efficient harmonics rejection is realized by harmonic restrain structure inside the quadrupling part and the filter between the quadrupling part and the output amplifier. According to the measured results, the output power reaches $20 \mathrm{dBm}$ when input power ranges from $-5 \mathrm{dBm}$ to $1 \mathrm{dBm}$ at an input frequency of $8.5 \mathrm{GHz}$. The harmonics rejections are larger than $40 \mathrm{dBc}$. The overall chip size is $3 \times 2.2 \mathrm{~mm}^{2}$.

Keywords: quadrupler, conversion gain, amplifier, harmonic, MMIC

Classification: Microwave and millimeter-wave devices, circuits, and modules

\section{References}

[1] K. Yuk and G. R. Branner: "Advances in active microwave frequency multipliers,” IEEE Int. Midwest Circuits Syst. Symp. (2011) 1 (DOI: 10.1109/ MWSCAS.2011.6026400).

[2] H. Okazaki and K. Araki: "A low-power Ku-band GaAs monolithic frequency quadrupler,” 2003 IEEE GaAs IC Symp. Dig. (2003) 255.

[3] K. Kamozaki, et al.: "A highly integrated Ka-band MMIC quadrupler," 2004 IEEE MTT-S International Microwave Symposium 1 (2004) 171 (DOI: 10. 1109/MWSYM.2004.1335835).

[4] P. Sandhiya, et al.: "A Ka band, low power dissipation, high spectral purity GaAs pHEMT MMIC X4 multiplier,” 2006 IEEE MTT-S International Microwave Symposium (2006) 1513 (DOI: 10.1109/MWSYM.2006.249599).

[5] T. Tokumitsu: "K-band and millimeter-wave MMICs for emerging commercial wireless applications," IEEE Trans. Microw. Theory Techn. 49 (2001) 2066 (DOI: 10.1109/22.963138).

[6] T. Masuda, et al.: “A high spectral purity GaAs PHEMT MMIC balanced 
frequency quadrupler," IEEE Gallium Arsenide Integrated Circuit (GaAs IC) Symposium (2003) 255 (DOI: 10.1109/GAAS.2003.1252406).

[7] K. Shirakawa, et al:: "A 15/60 GHz one-stage MMIC frequency quadrupler," IEEE 1996 Microwave and Millimeter-Wave Monolithic Circuits Symposium, Digest of Papers (1996) 35 (DOI: 10.1109/MCS.1996.506298).

[8] M. Abata, et al.: "A V-band MMIC frequency quadrupler with active input matching," 2015 International Conference on Electrical and Information Technologies (ICEIT) (2015) 134 (DOI: 10.1109/EITech.2015.7162968).

[9] HMC370, a SMT GaAs HBT MMIC $\times 4$ active frequency multiplier of ADI (Analog Devices, Inc.).

[10] HMC695, a SMT GaAs HBT MMIC $\times 4$ active frequency multiplier of ADI (Analog Devices, Inc.).

[11] CHX2092a99F, a GaAs MMIC $\times 4$ active frequency multiplier of UMS (United Monolithic Semiconductors).

\section{Introduction}

Frequency multiplier are widely used in millimeter-wave (MMW) system due to their advantages of highly spectral purity and stability [1,2]. With the increasing requirement of compact circuits, Microwave Monolithic Integrated circuits (MMIC) frequency multipliers up to hundreds $\mathrm{GHz}$ have been studied. However, the dynamic range and harmonic rejection of high-order MMIC multiplier (i.e. Quadrupler) is still unsatisfied in millimeter-wave band.

A wide variety of methods have been used in the past to perform frequency quadrupling The methods can be catalog into active or passive, single-ended or balanced, and cascading or single-stage. Compared with passive multipliers, the active multipliers have the advantage of providing possible conversion gain. Cascading two frequency doublers is also generally used [3, 4], but the suppression of the second harmonic is usually unsatisfactory. Balanced configuration is considered because it provides an efficient rejection of the fundamental and odd-harmonic frequency, and it is appropriate for implementation of a balanced $\mathrm{VCO}$ at the input $[3,4,5,6]$. As a basic topology of multiplier MMIC, single frequency multiplier circuit structure is relatively simple, flexible and convenient design which is often used by designers [7], and cascading amplifier and band-pass filter can effectively compensate for the lack of conversion gain and harmonic suppression.

In this paper, the design based on $0.15 \mu \mathrm{m}$ GaAs PHEMT process and performance of a frequency quadrupler comprised of a one-stage input power amplifier, a one-stage single-ended frequency quadrupler, a microstrip high-pass filter, and a three-stage output power amplifier is presented. Due to the chosen configuration, the quadrupler achieves high conversion gain and high output power level with, at the same time, excellent suppression of undesired harmonics. Furthermore, the size of the MMIC is limited to $3 \times 2.2 \mathrm{~mm}^{2}$.

\section{Circuit design}

The block diagram and chip layout is shown in Fig. 1. The configuration consists of an $8.5 \mathrm{GHz}$ one-stage input power amplifier, a $8.5-34 \mathrm{GHz}$ quadrupling part, a 
high-pass filter, and a $34 \mathrm{GHz}$ three-stage output power amplifier. The input power amplifier supplies exactly the proper power that the quadrupling part needs to realize relatively low conversion loss. Furthermore, a high-pass filter is designed between the quadrupling part and the output power amplifier. Thus, the MMIC performs well in conversion gain and unwanted harmonics' suppression.

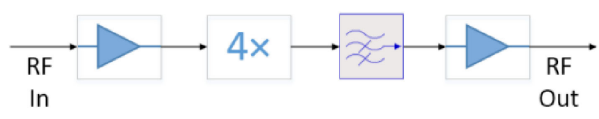

(a)

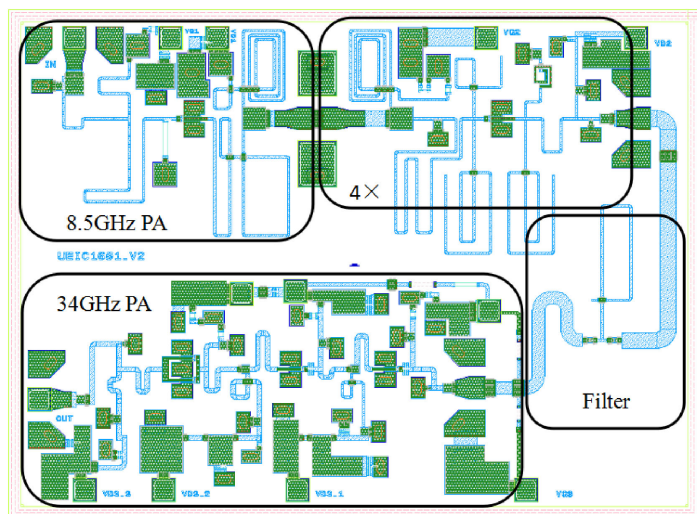

(b)

Fig. 1. Block diagram (a) and chip layout (b) of the MMIC quadrupler.

\subsection{Quadrupling part}

This part is the core of the whole design, whose schematic diagram is shown in Fig. 2. WIN's PHEMT standard $2 \times 50 \mu \mathrm{m}$ MS transistor working in class-B condition is used in this part. In order to reduce the dc power consumption and generate the fourth harmonic signal the transistor is biased near the pinch off region [8], where the non-linearity of transconductance is used for frequency multiplying.

The length of T2, T3, and T7 is shown in (1). $\lambda_{0}$ is the wavelength of the fundamental, and $\mathrm{f}_{0}$ is the frequency of the fundamental. As shown in Fig. 2., at the gate of the transistor, open-stub for $4 \times \mathrm{f}_{0}$ and open-stub for $2 \times \mathrm{f}_{0}(\mathrm{~T} 2$ and $\mathrm{T} 3)$

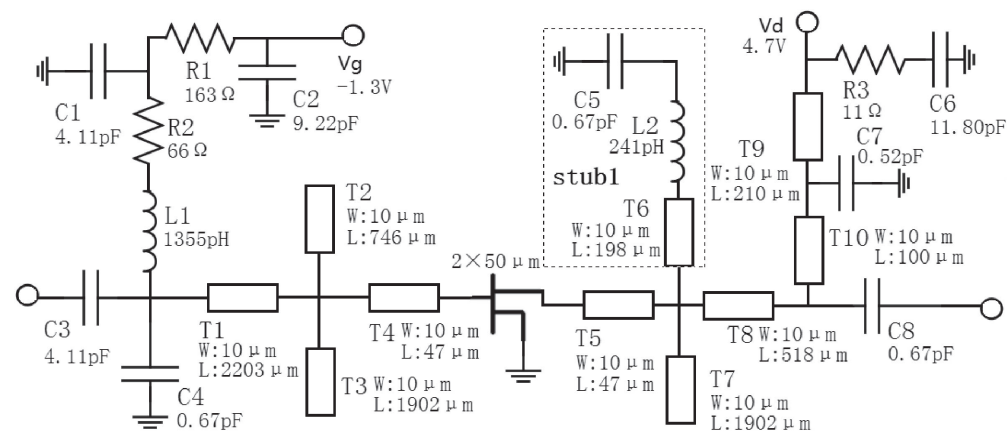

Fig. 2. Schematic diagram of the EM-simulation of the quadrupling part. 
supply a short circuit for the fourth harmonic and the second harmonic respectively to ensure the input signal avoiding interference of even harmonics. Two stubs (stub1 and T7) at the drain of the transistor are designed to suppress second harmonic, sixth harmonic and fundamental, third harmonic, fifth harmonic respectively. Considering that $\lambda_{0} / 4$ is too long, lumped elements instead of a long transmission line are used at stub1 [2, 7]. In order to improve stability of the circuit, resistors R1, R2 and R3 are used at the dc bias stub. Input and output matching structures are thoroughly investigated to bring the least transition loss for fundamental and the fourth harmonics respectively.

$$
l_{3}=l_{7}=\lambda_{0} / 8 \quad l_{2}=\lambda_{0} / 16
$$

Fig. 3. Shows the output spectrum when input power of this part is $12.42 \mathrm{dBm}$ at $8.5 \mathrm{GHz}$. According to the simulation result, undesired harmonic suppression can reach $13.3 \mathrm{dBc}$. The rejection of the third harmonic is about $27 \mathrm{dBc}$.

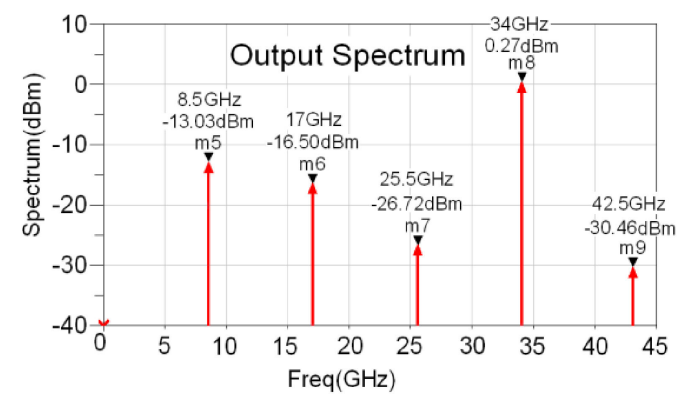

Fig. 3. Simulation result of the quadrupling part.

\section{2 $8.5 \mathrm{GHz}$ drive power amplifier}

Fig. 4. Shows the schematic diagram of the $8.5 \mathrm{GHz}$ drive power amplifier. In order to drive the quadrupling part, standard $2 \times 75$ um MS transistor is used in this part. The input-matching network has transmission lines T1, T2 and T3. Capacitor C3 can expand bandwidth to a certain extent, and also takes part in the input-matching. $\mathrm{R} 2$ and $\mathrm{C} 4$ can reduce low frequency gain and improve stability at low frequencies. R1 is a current limiting resistor. At drain, T4 and L play the role of outputmatching. R3 brings down the gain, but can guarantee the stability.

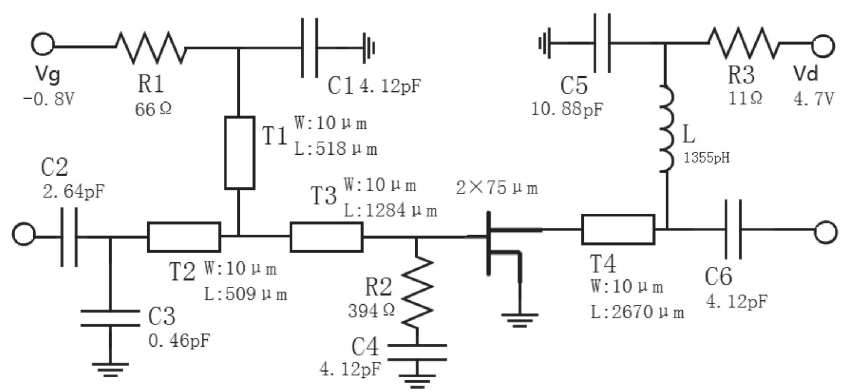




\section{3 $34 \mathrm{GHz}$ output power amplifier}

Due to conversion loss of the second part, the output power of the quadrupler is about $-1 \mathrm{dBm}$. Thus, an output power amplifier is designed to improve the conversion gain of the whole MMIC and so enough output power that the application of the quadrupler is much more convenient. The output power amplifier not only increases the output power of the quadrupler, but also plays a role in rejection of other harmonics.

Schematic diagram of the three-stage $34 \mathrm{GHz}$ output power amplifier is presented in Fig. 5. Gate width of three transistors are respectively $2 \times 50 \mathrm{um}$, $2 \times 50 \mathrm{um}, 4 \times 50 \mathrm{um}$. Matching structures and dc bias circuits of each stage are designed quite similar. L-type microstrip matching structure is adopted at each gate and drain of the transistors. In order to reduce gain and improve the stability, a series resistance is added to the gate of each transistor. Capacitors are cascaded between stages in order to isolate biases. Resistors and capacitors used in the bias structures reduce the low frequency gain and improve the stability at low frequencies.

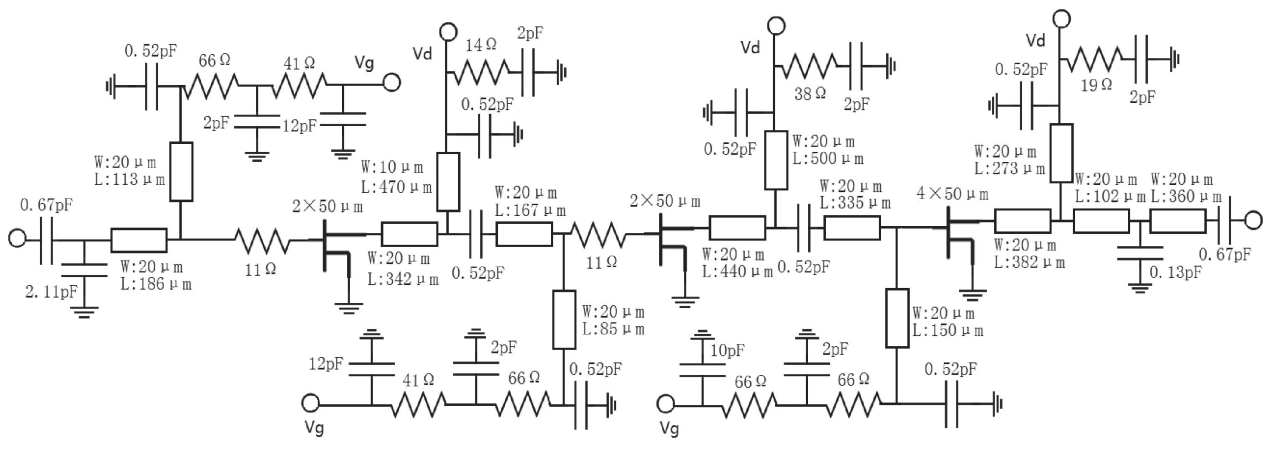

Fig. 5. Schematic diagram of the part of $34 \mathrm{GHz}$ output power amplifier.

\subsection{High-pass filter}

In order to realize higher suppression of lower harmonics, a high-pass filter is designed between the quadrupling part and the output power amplifier. Its schematic diagram is shown in Fig. 6. The filter is structured by transmission line cascaded with two capacitance paralleled with an open stub. Function of highpass is formed by the capacitance, and the open stub shown in Fig. 6. generates a resonance point of the third harmonic. Simulation result of this part is shown in Fig. 7.

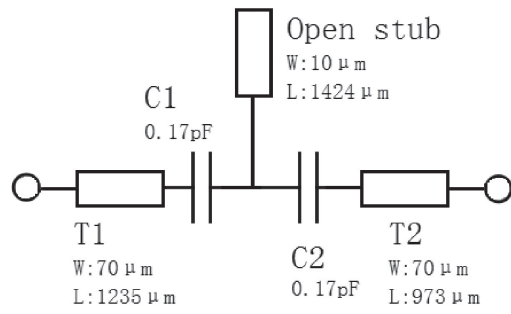




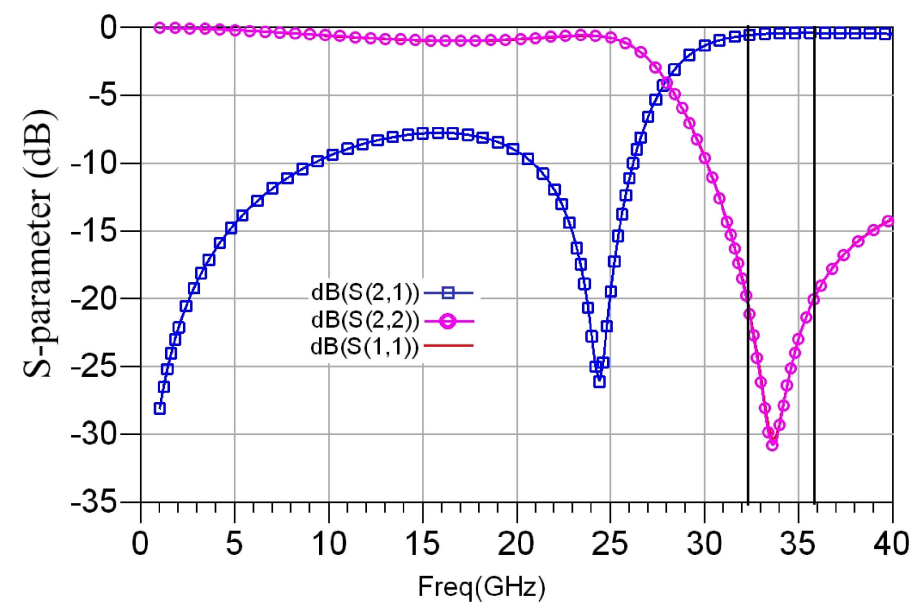

Fig. 7. Simulation result of the high-pass filter.

\section{Measured MMIC performance}

The test board shown in Fig. 8. is designed. The testing work is done on probe station for that RF signal is still added and received through GSG probes.

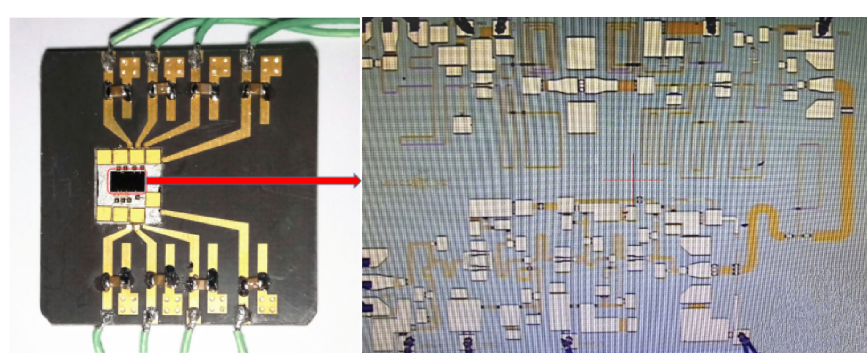

Fig. 8. Test board and the picture of the MMIC.

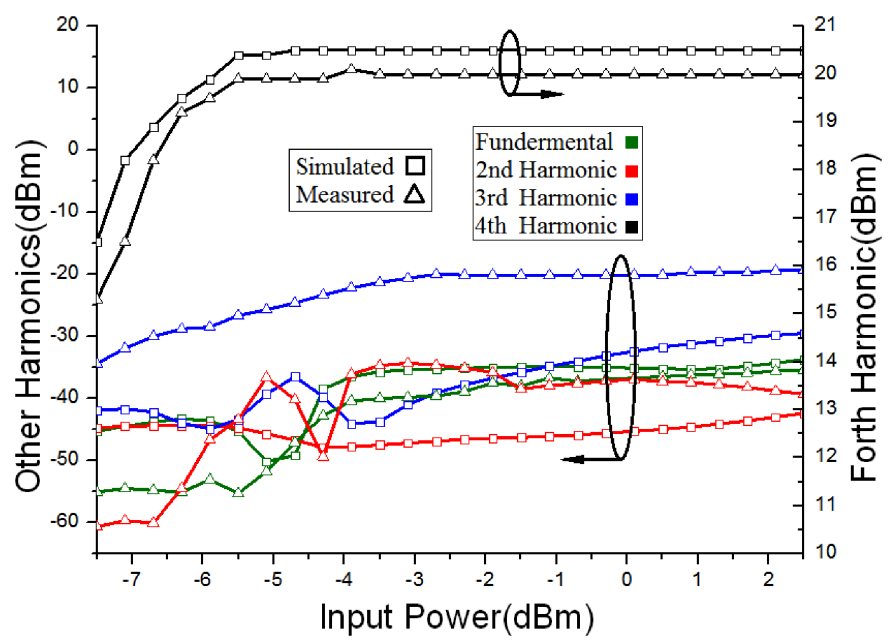

Fig. 9. Measured output power of the quadrupler in comparison with the simulation result as a function of input power at an input frequency of $8.5 \mathrm{GHz}$.

Fig. 9. presents the measured output power of each harmonic (from fundamental to the forth harmonic) of the quadrupler in comparison with the simulation result as a function of input power at an input frequency of $8.5 \mathrm{GHz}$. The quadrupler delivers $20 \mathrm{dBm}$ when it gets saturated at an input power of about 
$-5 \mathrm{dBm}$. Compared with simulation result, measured result is a little worse. We can judge from the graph that the rejection of fundamental is beyond $55 \mathrm{dBc}$, the rejection of the second harmonic is beyond $54 \mathrm{dBc}$, and for the worst, the rejection of the third harmonic is beyond $40 \mathrm{dBc}$. The total working current of the quadrupler is less than $150 \mathrm{~mA}$. The quadrupler performs quite similar when input frequency ranges from $8.4 \mathrm{GHz}$ to $8.6 \mathrm{GHz}$. The flatness of output power of the quadrupler is $0.5 \mathrm{dBpp}$ from $33.6 \mathrm{GHz}$ to $34.4 \mathrm{GHz}$.

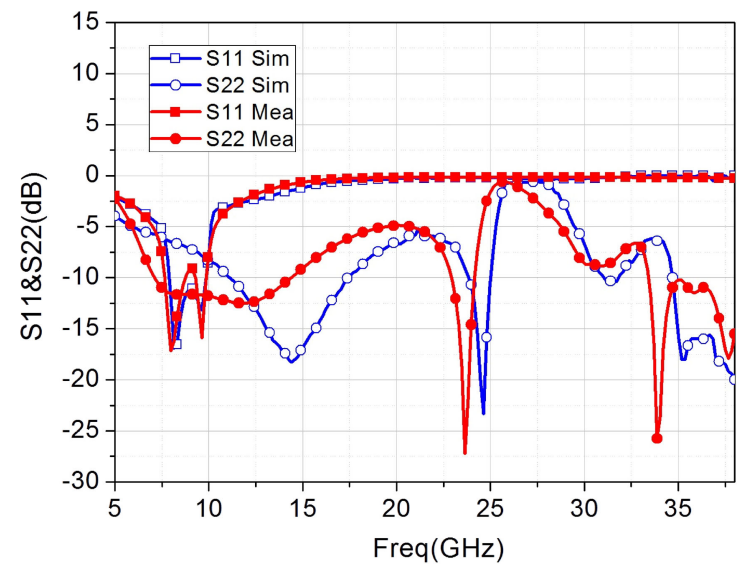

Fig. 10. Measured $S_{11}$ and $S_{22}$ of the quadrupler in comparison with the simulation results.

Fig. 10. shows the measured result of $S_{11}$ and $S_{22}$ compared with the simulation ones. We can judge with this result that the measured S-parameter offsets down from the simulation one. The offset increases with the increase of frequency. It's obvious that the offset is about $2 \mathrm{GHz}$ at $25.5 \mathrm{GHz}$ which is the frequency of third harmonic. This conclusion may cause offset of the simulation result of the filter shown in Fig. 7. Which is the reason of the distinguish of output harmonics between test and simulation shown in Fig. 9.

Table I. Summarizes some of the characteristics of the published MMIC quadruplers or the products of major microwave or RF corporations. The perform-

Table I. MMIC quadruplers performances.

\begin{tabular}{|c|c|c|c|c|c|c|c|}
\hline Ref. & Type & $\begin{array}{c}\text { Pout } \\
(\mathrm{dBm})\end{array}$ & $\begin{array}{c}\text { Freqout } \\
(\mathrm{GHz})\end{array}$ & Rejct. $(\mathrm{dBc})$ & $\begin{array}{c}\text { Conv.G. } \\
(\mathrm{dB})\end{array}$ & $\begin{array}{c}\text { Size } \\
\left(\mathrm{mm}^{2}\right)\end{array}$ & $\begin{array}{c}\mathrm{P}_{\mathrm{DC}} \\
(\mathrm{mW})\end{array}$ \\
\hline$[2]$ & 2 sing. $\times 2$ & 1.4 & $12-12.8$ & - & 4.4 & 1.69 & 35 \\
\hline$[3]$ & 2 bal. $\times 2$ & 19.4 & $36-40$ & 30 & 16.4 & 2.76 & 1596 \\
\hline$[4]$ & 2 bal. $\times 2$ & 0 & $27-34$ & 30 & -5 & 6.75 & 163 \\
\hline$[6]$ & bal. $\times 4$ & -2.5 & 30 & 30 & -7.5 & 3 & 54 \\
\hline$[7]$ & sing. $\times 4$ & -5 & 59 & - & -5 & 2.38 & \\
\hline$[8]$ & sing. $\times 4$ & 6.9 & $56-64$ & $40($ for & 6.9 & - & - \\
\hline$[9]$ & $\times 4$ & 0 & $14.4-16.4$ & 22 & $-5-15$ & Packaged & 275 \\
\hline$[10]$ & $\times 4$ & 7 & $11.4-13.2$ & 25 & $2-17$ & Packaged & 300 \\
\hline$[11]$ & $2 \times 2$ & 11 & $36-40$ & 15 & -1 & 2.25 & 280 \\
\hline$[$ th] & sing. $\times 4$ & $\mathbf{2 0}$ & $32.4-35.6$ & $\mathbf{4 0}$ & $\mathbf{1 9 - 2 5}$ & 6.6 & 705 \\
\hline
\end{tabular}


ances of the quadrupler designed in this paper is mentioned at the bottom of the table for comparison. To my knowledge, such significant performance in conversion gain and undesired harmonic suppression of this work has never shown up in former works.

\section{Conclusion}

The design of a Ka-band GaAs MMIC quadrupler with high conversion gain and efficient rejection of undesired harmonics is discussed in this publication and the chosen configuration realizes a remarkable performance as shown in the measured result. With a chip size of $3 \times 2.2 \mathrm{~mm}^{2}$, the MMIC performs that the conversion gain ranges from $25 \mathrm{~dB}$ to $19 \mathrm{~dB}$ and correspondingly the output power reaches $20 \mathrm{dBm}$ when input power ranges from $-5 \mathrm{dBm}$ to $1 \mathrm{dBm}$ at an input frequency of $8.5 \mathrm{GHz}$. Meanwhile, the rejection of fundamental is $55 \mathrm{dBc}$ and rejection of unwanted harmonic is $54 \mathrm{dBc}$ for the second harmonic, and $40 \mathrm{dBc}$ for the third at an input power of $-2 \mathrm{dBm}$, frequency of $8.5 \mathrm{GHz}$. 